\title{
THE COMMUTATOR SUBGROUP MADE ABELIAN
}

\author{
JOEL M. COHEN ${ }^{1}$
}

\begin{abstract}
A theorem on covering spaces is proved which yields the following information about a group $\pi$, its commutator subgroup $\pi^{\prime}$ and their abelianizations: If $\pi^{a b} \cong Z_{p^{n}}$, a cyclic group of order a power of the prime $p$, then $\pi^{a b}=p \pi^{\prime a b}$. Hence if $\pi$ is also finitely generated, then $\pi^{\prime a b}$ is finite of order prime to $p$.
\end{abstract}

The purpose of this note is to prove the following theorem and some related results:

THEOREM 1. Let $X$ be a connected $\mathrm{CW}$ complex with $H_{1}(X) \cong Z_{p^{n}}$. Let $X^{\prime}$ be the normal $p^{n}$-fold covering space of $X$ with transformation group $Z_{p^{n}}$. Then $H_{1}\left(X^{\prime}\right)$ is p-divisible. In particular, if $\pi_{1} X$ is finitely generated, then $\pi_{1} X^{\prime}$ is also so $H_{1}\left(X^{\prime}\right)$ is finite of order prime to $p$.

Notation. If $\pi$ is a group, $\pi^{\prime}=[\pi, \pi]$ is the commutator subgroup and $\pi^{a b}=\pi / \pi^{\prime}$ is its abelianization.

An immediate corollary is

THEOREM 2. If $\pi^{a b} \cong Z_{p^{n}}$, a cyclic group of order a power of the prime $p$, then $\pi^{\prime a b}$ is p-divisible; i.e. $\pi^{\prime a b}=p \pi^{\prime a b}$.

Theorem 2 follows from Theorem 1 by observing that the EilenbergMac Lane space $K\left(\pi^{\prime}, 1\right)$ is the $p^{n}$-fold covering space of $K(\pi, 1)$ and $H_{1}(K(\pi, 1))=\pi^{a b}, H_{1}\left(K\left(\pi^{\prime}, 1\right)\right)=\pi^{\prime a b}$.

The proof of Theorem 1 is based on the homology Serre Spectral Sequence of the fibration $X^{\prime} \rightarrow X \rightarrow K\left(Z_{p^{n}}, 1\right): E_{* *}^{2}=H_{*}\left(Z_{p^{n}} ; H_{*}\left(X^{\prime}\right)\right)$ (local coefficients based on the action of $Z_{p^{n}}$ on $X^{\prime}$ ) converging to $H_{*}(X)$ (simple $Z$-coefficients).

Because it is a first quadrant spectral sequence there is an exact sequence

$$
E_{2,0}^{2} \rightarrow E_{0,1}^{2} \rightarrow H_{1}(X) \rightarrow E_{1,0}^{2} \rightarrow 0 \text {. }
$$

Received by the editors March 14, 1972.

AMS (MOS) subject classifications (1970). Primary 20F35, 55A10; Secondary $18 \mathrm{H} 10,55 \mathrm{~B} 25$.

Key words and phrases. Abelianization, commutator subgroup, covering space, homology of groups.

${ }^{1}$ This work was partially supported by a grant from the National Science Foundation.

(c) American Mathematical Society 1973 
But $E_{s, 0}^{2}=H_{s}\left(Z_{p^{n}} ; H_{0}\left(X^{\prime}\right)\right)=H_{s}\left(Z_{x^{n}}\right)$ which is $Z_{p^{n}}$ for $s=1$ and 0 for $s=2$. Since $H_{1}(X) \rightarrow H_{1}\left(Z_{p^{n}}\right)$ is an isomorphism, we conclude that

$$
H_{0}\left(Z_{p^{n}} ; H_{1}\left(X^{\prime}\right)\right)=E_{0,1}^{2}=0 .
$$

The theorem is proved once we show:

Proposition. If $G$ is a finite p-group and $H_{0}(G ; M)=0$ for some $G$ module $M$, then $M$ is p-divisible.

Proof. $\quad H_{0}(G ; M)=M \mid I M$ where $I=\operatorname{ker} \varepsilon: Z[G] \rightarrow Z$ is the augmentation. So $H_{0}(G ; M)=0$ means $M=I M$. The proposition will be proved if we can show that for some integer $N, I^{N} \subset p I$, whence $M=I^{N} M \subset p I M=p M$. This is equivalent to showing that $J=I \otimes Z_{p}=\operatorname{ker} \varepsilon \otimes Z_{p}$ is nilpotent. This is well known [1, p. 703] but for completeness we prove it here for the case $G=Z_{p^{n}}$ : Let $t$ be the generator of $Z_{p^{n}}$ (written multiplicatively). $t^{p^{n}}=1$. Then $J=(t-1) Z_{p}[G] . \quad J^{p^{n}}=(t-1)^{p^{n}} Z_{p}[G]$. But modulo $p$, $(t-1)^{p^{n}} \equiv\left(t^{p^{n}}-1\right)=0$ so $J^{p^{n}}=0$.

\section{REFERENCE}

1. Dock Sang Rim, Modules over finite groups, Ann. of Math. (2) 69 (1959), 700-712. MR 21 \#3474.

Department of Mathematics, University of Pennsylvania, Philadelphia, PenNSylVania 19104 